\title{
GENERATION OF SUMMAND ABSORBING SUBMODULES
}

\author{
ZUR IZHAKIAN, MANFRED KNEBUSCH, AND LOUIS ROWEN
}

\begin{abstract}
An $R$-module $V$ over a semiring $R$ lacks zero sums (LZS) if $x+y=0 \Rightarrow$ $x=y=0$. More generally, a submodule $W$ of $V$ is "summand absorbing", if $\forall x, y \in V$ : $x+y \in W \Rightarrow x \in W, y \in W$. These relate to tropical algebra and modules over idempotent semirings, as well as modules over semirings of sums of squares. In previous work, we have explored the lattice of summand absorbing submodules of a given LZS module, especially those that are finitely generated, in terms of the lattice-theoretic Krull dimension. In this note we describe their explicit generation.
\end{abstract}

\section{Contents}

1. Introduction

2. Generating SA-submodules by use of additive spines

3. Halos and additive spines in $R$-modules

4. The posets $\mathrm{SA}(V), \Sigma \mathrm{SA}(V)$ and $\Sigma_{f} \mathrm{SA}_{f}$ in good cases

References

\section{INTRODUCTION}

Semirings, initiated by Costa [1] and exposed by Golan [2], have played an increasing role recently due to increased interest to tropical algebra which involves the max-plus algebra and related constructions. Another important example is the set of positive elements in an ordered ring. Both of these examples lack zero sums (termed "zero sum free" in [2]) in the following sense: An $R$-module $V$ over a semiring $R$ lacks zero sums (abbreviated LZS), if

$$
\forall x, y \in V: x+y=0 \Rightarrow x=y=0 .
$$

As noted in [4, Proposition 1.8], any module over an idempotent semiring is LZS, yielding a large assortment of examples. Furthermore, by [4, Examples 1.6] being LZS is closed under submodules, direct products, and modules $\operatorname{Fun}(S, V)$ of functions from a set $S$ to a module $V$. Thus, examples include the max-plus algebra, function semirings, polynomial semirings and Laurent polynomial semirings over idempotent semirings, and the "boolean semifield" $\mathbb{B}=\{-\infty, 0\}$ (and thus subalgebras of algebras that are free modules over $\mathbb{B}$ ). This shows that our results pertain to "F $\mathbb{F}_{1}$-geometry."

Date: January 14, 2019.

2010 Mathematics Subject Classification. Primary 14T05, 16D70, 16Y60 ; Secondary 06F05, 06F25, $13 \mathrm{C} 10,14 \mathrm{~N} 05$.

Key words and phrases. Semiring, lacking zero sums, direct sum decomposition, free (semi)module, summand absorbing submodule, halo, additive spine.

File name: SAsubmodulesGeneration15. 
Continuing the theory from [4], we called a submodule $W$ of $V$ summand absorbing (abbreviated SA) in $V$, if

$$
\forall x, y \in V: x+y \in W \quad \Rightarrow \quad x \in W, y \in W
$$

we then call $W$ an SA-submodule of $V$. An SA-left ideal of a semiring $R$ is an SAsubmodule of $R$.

SA-submodules were the main subject of investigation in [5], largely because of their nice lattice-theoretic properties. The objective of [5] was to continue to develop the theory of SAmodules over semirings, along the lattice-theoretic lines of classical module theory (especially the Noetherian theory), with the goal of paralleling the Wedderburn-Miller-Remak-KrullSchmidt and Jordan-Hölder theorems.

Given an $R$-module $V$ and a set $S$ of generators of $V$, we detect a new set $T$ of generators of $V$, which is "small" in some sense if $S$ is "small", and gives us sets of generators of all SA-submodules $W$ of $V$ in a coherent way. Here is an instance.

Definition 1.1. A set of generators $T$ of $V$ is $\boldsymbol{S A}$-adapted, if every $S A$-submodule $W$ of $V$ is generated by the set $W \cap T$.

We obtain a reasonable SA-adapted set of generators $T$ from a given set of generators $S$ by employing the so-called additive spine $M$ of a module:

Definition 1.2. Assume that $S$ is a subset of $V$.

a) The halo $\widetilde{S}$ of $S$ in $V$ is the set of all $v \in V$ such that there exist $\lambda, \mu \in R$ with $\lambda v \in S$ and $\mu \lambda v=v$.

b) $S$ is called an additive spine of the $R$-module $V$, if $V$ is additively generated by $\widetilde{S}$, which we denote as $V=\sum^{\infty} \widetilde{S}$.

Thus the additive spines of $R$ are of $R$ considered as a left $R$-module.

In the special case that both $M$ and $S$ are finite it will turn out that also $T$ is finite, and so all SA-submodules $W$ of $V$ are generated by at most $|T|$ elements.

Example 1.3. If $V$ has a finite $S A$-adapted set of generators, then $V$ is SA-artinian (as defined in [5, Definition 1.4]).

One main general result:

Theorem 3.2. Assume that $S$ is an additive spine of an $R$-module $V$. Then every $S A$ submodule $W$ of $V$ is generated by $W \cap S$, and moreover $W \cap S$ is an additive spine of $W$.

For semirings, this specializes in $₫ 2$ to:

Theorem 2.8. Assume that $S$ is a set of generators of an $R$-module $V$, and $M$ is an additive spine of $R$. Then any $S A$-submodule $W$ of $V$ is generated by the set $W \cap(M S)$.

Consequently, if $V$ is generated by $n$ elements, then every SA-submodule of $V$ is generated by $m n$ elements, where $m=|M|$ is independent of $n$. An application to matrices is given in Theorem 2.14, and more generally to monoid semirings in Theorem 2.17 .

Section 4 focuses on finite generation in terms of finite additive spines.

\section{Generating SA-Submodules by use of AdDitive spines}

Throughout this paper, $R$ is a semiring (with $1=1_{R}$ ), and $V$ is a (left) module (sometimes called "semimodule") over $R$; i.e., $(V,+)$ is a monoid satisfying the familiar module axioms as well as $r 0_{V}=0_{R} x=0_{V}$ for all $r \in R, x \in V$. The zero submodule $\left\{0_{V}\right\}$ is usually written as 0 . 
We first state basic facts about additive spines of $R$, and give basic examples.

Definition 2.1. (Special case of Definition 1.2). Given a subset $M$ of $R$.

a) We define the set

$$
\widetilde{M}:=\{x \in R \mid \exists y, z \in R: y x \in M, z y x=x\},
$$

which we call the halo of $M$ in $R$.

b) If the halo $\widetilde{M}$ additively generates $R$, i.e., $R=\sum^{\infty} \widetilde{M}$, we call $M$ an additive spine of $R$.

We state some facts about halos which are immediate consequences of Definition 2.1.a.

\section{Remarks 2.2.}

i) $M \subset \widetilde{M}$ for any set $M \subset R$.

ii) If $M \subset N \subset R$, then $\widetilde{M} \subset \tilde{N}$.

iii) If $\left(M_{i} \mid i \in I\right)$ is a family of subsets of $R$, then

$$
\left(\bigcup_{i \in I} M_{i}\right)^{\sim}=\bigcup_{i \in I} \widetilde{M}_{i}
$$

iv) $\left\{0_{R}\right\}^{\sim}=\left\{0_{R}\right\}$ and $\left(M \backslash\left\{0_{R}\right\}\right)^{\sim}=\widetilde{M}$.

Due to the last remark we may assume in any study of halos that $0_{R} \in M$ or $0_{R} \notin M$, whatever is more convenient.

Here are perhaps the most basic examples of halos deserving interest.

Example 2.3. Let $M=\left\{1_{R}\right\}$. Then $\widetilde{M}$ is the set of left invertible elements of $R$. Indeed, if $x \in \widetilde{M}$, then there exists $y \in R$ with $y x=1_{R}$. Conversely, if $x$ is left-invertible there exists $y \in R$ with $y x=1_{R}$, and so $x y x=x$, which proves that $x \in \widetilde{M}$.

Example 2.4. Let $M=\{e\}$ with e an idempotent of $R$. If $x \in \widetilde{M}$, then there exist $y, z \in R$ with $y x=e, z e=x$. It follows that $x e=x$, yielding the von Neumann condition $x y x=x$, cf. 3]. Conversely, if $y x=e$ and $x y x=x$, then clearly $x \in \widetilde{M}$. This proves that

$$
\{e\}^{\sim}=\{x \in R \mid \exists y \in R: y x=e, x y x=x\} .
$$

Let $\operatorname{Id}(R)$ denote the set of all idempotents of $R$. Starting from Example 2.4, we obtain the following fact.

Proposition 2.5. If $R$ is any semiring, then

$$
\operatorname{Id}(R)^{\sim}=\{x \in R \mid \exists y \in R: x y x=x\} .
$$

Proof. $\operatorname{Id}(R)^{\sim}$ is the union of the sets $\{e\}^{\sim}$ with $e$ an idempotent of $R$ (cf. Remark 2.2.iii). Thus it is clear from Example 2.4 that for every $x \in \operatorname{Id}(R)^{\sim}$ there exists some $y \in R$ with $x y x=x$. Conversely, if $x y x=x$, then $y x \cdot y x=y x$, and so $e:=y x$ is an idempotent of $R$. Moreover $x e=x$, and so $x \in\{e\}^{\sim}$.

We state an immediate consequence of this proposition.

Corollary 2.6. For any subset $M$ of $R$ we have

$$
[M \cap \operatorname{Id}(R)]^{\sim}=\{x \in \widetilde{M} \mid \exists y \in R: x y x=x\},
$$

and $\widetilde{M}$ is the disjoint union of this set and $[M \backslash \operatorname{Id}(R)]^{\sim}$. 
The set $[M \cap \operatorname{Id}(R)]^{\sim}$ may be regarded as the "easy part" of the halo $\widetilde{M}$.

Notation 2.7. Given (nonempty) subsets $A, B$ of $R$, we denote the set of all products ab with $a \in A, b \in B$ by $A B$ (or $A \cdot B$ ). Similarly, if $A \subset R$ and $X \subset V$, then $A X$ denotes the set of products ax with $a \in A, x \in X$. Furthermore, $\sum^{\infty} A$ and $\sum^{\infty} X$ denote the set of all finite sums of elements of $A$ in $R$ and of $X$ in $V$ respectively. Admitting also the empty sum of elements of $A$ or $X$, we always have $0_{R} \in \sum_{\infty}^{\infty} A, 0_{V} \in \sum^{\infty} X$. If necessary, we write more precisely $\sum_{R}^{\infty} A$ and $\sum_{V}^{\infty} X$ instead of $\sum^{\infty} A$ and $\sum^{\infty} X$.

In this notation, a set $S \subset V$ generates the $R$-module $V$, if $V=\sum^{\infty} R S$.

We are ready for a central result in this note. $\mathrm{SA}(V)$ denotes the poset consisting of all SA-submodules of $V$.

Theorem 2.8. Assume that $S$ is a set of generators of a (left) $R$-module $V$, and $M$ is an additive spine of $R$. Then any $S A$-submodule $W$ of $V$ is generated by the set $W \cap(M S)$.

Proof. Since $V=\sum^{\infty} R S$ and $R=\sum^{\infty} \widetilde{M}$, we have $V=\sum^{\infty} \widetilde{M} S$.

Let $w \in W, w \neq 0$, be given. Then

$$
w=\sum_{i=1}^{n} x_{i} s_{i}
$$

with $n \in \mathbb{N}, s_{i} \in S, x_{i} \in \widetilde{M}$. Since $W$ is in $\mathrm{SA}(V)$, it follows that

$$
x_{i} s_{i} \in W \quad \text { for } 1 \leq i \leq n .
$$

Now choose $y_{i}, z_{i} \in R$ such that $m_{i}:=y_{i} x_{i} \in M$ and $x_{i}=z_{i} m_{i}$. Then

$$
y_{i}\left(x_{i} s_{i}\right)=m_{i} s_{i} \in W \cap(M S)
$$

and

$$
z_{i} m_{i} s_{i}=z_{i} y_{i} x_{i} s_{i}=x_{i} s_{i}
$$

From (A) we obtain that

$$
w=\sum_{i=1}^{n} z_{i}\left(m_{i} s_{i}\right) .
$$

We conclude from $(\overline{\mathrm{B}})$ and $(\mathrm{C})$ that $W \cap(M S)$ generates $W$.

Corollary 2.9. Assume that $R$ has a finite additive spine $M$ and $V$ has a finite set of generators $S$. Then every $S A$-submodule $W$ of $V$ is finitely generated, more precisely, generated by at most $|M| \cdot|S|$ elements (independent of the choice of $W !$ ).

Theorem 2.10. Assume that $V$ is a module over a semiring $R$ that is additively generated by the set of its left invertible elements. Then every set of generators $S$ of $V$ is SA-adapted.

Proof. We read off from Example 2.3 that $\left\{1_{R}\right\}$ is an additive spine of $R$. So by Theorem 2.8 every SA-submodule $W$ of $V$ is generated by $W \cap S=W \cap\left(1_{R} S\right)$.

We take a look at additive spines of matrix semirings. 
Example 2.11. Assume that $C$ is a semiring that is additively generated by $\left\{1_{C}\right\}$,

$$
C=\sum^{\infty}\left\{1_{C}\right\}
$$

In other terms, the unique homomorphism $\varphi: \mathbb{N}_{0} \rightarrow C$ with $\varphi(1)=1_{C}$ is surjective. Then the semiring

$$
R=M_{n}(C)=\sum_{i, j=1}^{n} C e_{i j}
$$

of $(n \times n)$-matrices with entries in $C$, and $e_{i j}$ the usual matrix units, has the additive spine

$$
D:=\left\{e_{11}, e_{22}, \ldots, e_{n n}\right\}
$$

Indeed, for every $j \in\{1, \ldots, n\}$

$$
\left\{e_{j j}\right\}^{\sim} \supset\left\{e_{i j} \mid 1 \leq i \leq n\right\},
$$

since $e_{j i} e_{i j}=e_{j j}, e_{i j} e_{j j}=e_{i j}$, and so $\widetilde{D}=\bigcup_{j}\left\{e_{j j}\right\}^{\sim}$ contains the set $E:=\left\{e_{i j} \mid 1 \leq i, j \leq n\right\}$ of all matrix units, which by the nature of $C$ generates $M_{n}(C)$ additively.

This example can be amplified to a theorem about additive spines in arbitrary matrix rings $M_{n}(A)$ by use of a general principle to "multiply" additive spines, which runs as follows:

Proposition 2.12. Assume that $R_{1}$ and $R_{2}$ are subsemirings of a semiring $R$, such that $R$ is additively generated by $R_{1} R_{2}$, i.e., $R=\sum^{\infty} R_{1} R_{2}$. Assume moreover that the elements of $R_{1}$ commute with those of $R_{2}$. Assume finally that $M_{i}$ is an additive spine of $R_{i}$. Let $\widetilde{M}_{i}$ denote the halo of $M_{i}$ in $R_{i}(i=1,2)$. Then $\widetilde{M}_{1} \widetilde{M}_{2}$ is contained in the halo $\left(M_{1} M_{2}\right)^{\sim}$ of $M_{1} M_{2}$ in $R$, and $M_{1} M_{2}$ is an additive spine of $R$.

Proof. Let $x_{i} \in \widetilde{M}_{i}(i=1,2)$ be given. We have elements $y_{i}, z_{i}$ of $R_{i}$ with $m_{i}:=y_{i} x_{i} \in M_{i}$ and $z_{i} m_{i}=x_{i}$. Now

$$
\left(y_{1} y_{2}\right)\left(x_{1} x_{2}\right)=\left(y_{1} x_{1}\right)\left(y_{2} x_{2}\right)=m_{1} m_{2}
$$

and

$$
\left(z_{1} z_{2}\right)\left(m_{1} m_{2}\right)=\left(z_{1} m_{1}\right)\left(z_{2} m_{2}\right)=x_{1} x_{2}
$$

This proves that $x_{1} x_{2} \in\left(M_{1} M_{2}\right)^{\sim}$. It follows that

$$
\left(\sum^{\infty} \widetilde{M}_{1}\right) \cdot\left(\sum^{\infty} \widetilde{M}_{2}\right)=R_{1} R_{2}
$$

and then that

$$
R=\sum^{\infty} R_{1} R_{2}=\sum^{\infty} \widetilde{M}_{1} \widetilde{M}_{2}
$$

Theorem 2.13. Assume that $R$ is the semiring of $(n \times n)$-matrices over a semiring $A$, so

$$
R:=M_{n}(A)=\sum_{i, j=1}^{n} A e_{i j}
$$

with the usual matrix units $e_{i j}$. Let $N$ be an additive spine of $A$. Then the set $M:=\bigcup_{i=1}^{n} N e_{i i}$, consisting of the diagonal matrices with entries in $N$, is an additive spine of $R$. 
Proof. Let $C$ denote the smallest subsemiring of $A, C=\left\{n \cdot 1_{A} \mid n \in \mathbb{N}\right\}$. We have seen that $R_{1}:=M_{n}(C)$ has the additive spine $D:=\left\{e_{i i} \mid 1 \leq i \leq n\right\}$ (Example 2.11). Let $R_{2}:=A \cdot 1_{R}$. This is the subsemiring of $R$ consisting of all matrices $a I$ with $a \in A$, where $I$ is the identity matrix. It has the additive spine $\mathbb{N} \cdot 1_{R_{2}}$. Now $R=\sum R_{1} R_{2}$, and the elements of $R_{1}$ commute with those of $R_{2}$. Thus, by Proposition $2.12, R$ has the additive spine $D \cdot\left(\mathbb{N} 1_{R_{2}}\right)=\bigcup_{i=1}^{n} \mathbb{N} e_{i i}$.

Recalling Theorem 2.8 we obtain

Theorem 2.14. Assume that $V$ is an $M_{n}(A)$-module, $A$ any semiring, and $S$ a system of generators of $V$. Assume furthermore that $N$ is an additive spine of $A$. Then any $S A$ submodule $W$ of $M_{n}(A)$ is generated by the set

$$
W \cap\left(\bigcup_{i=1}^{n} N e_{i i}\right)=\bigcup_{i=1}^{n} W \cap\left(N e_{i i}\right) .
$$

If $N$ is finite, then $W$ can be generated by at most $n \cdot|N|$ elements.

The proof of Theorem 2.13 can be seen in a much wider context, as we explain now.

Definition 2.15. Let $S=(S, \cdot)$ be a multiplicative monoid. We call a subset $T$ of $S$ a monoid spine of $S$, if for any $s \in S$ there exist $s_{1}, s_{2} \in S$ such that $t:=s_{1} s \in T$ and $s_{2} t=s$.

Given any semiring $A$ and monoid $S=(S, \cdot)$, we denote, as usual, the monoid-semiring of $S$ over $A$ by $A[S]$, which is the free $A$-module with base $S \backslash\{0\}$.

In the case that the monoid $S$ is without zero, i.e., $S$ does not contain an absorbing element $0,(0 \cdot S=S \cdot 0=0$ for all $s \in S)$, the elements $x$ of $R:=A[S]$ are the formal sums

$$
x=\sum_{s \in S} a_{s} s,
$$

with coefficients $a_{s} \in A$ uniquely determined by $x$, only finitely many non-zero. The multiplication is determined by the rule $(a s) \cdot(b t)=(a b)(s t)$ for $a, b \in A, s, t \in S$. Identifying $a=a \cdot 1_{S}, s=1_{A} \cdot s$, we regard $A$ as a subsemiring of $R$ and $S$ as a submonoid of $(R, \cdot)$.

If the monoid $S$ has a zero $0=0_{S}$, i.e., is pointed, we take for $R=A[S]$ the free $A$ module with base $S \backslash\{0\}$ and multiplication rule $(a s) \cdot(b t)=(a b)(s t)$ if $s t \neq 0,(a s)(b t)=0$ otherwise. Now the nonzero elements of $R=A[S]$ are formal sums $\sum_{s \neq 0} a_{s} s$. We identify again $a=a \cdot 1_{S}, s=1_{A} \cdot s$ for $s \in S \backslash\{0\}$, and now also $0=0_{A}$. Then again $A$ becomes a subsemiring of $R$ and $S$ a submonoid of $(R, \cdot)$. We have $R=\sum^{\infty} A S$ in both cases.

Example 2.16. The matrix semiring $M_{n}(A)$ coincides with $A[S]$, where $S$ is the monoid $\left\{e_{i j} \mid 1 \leq i, j \leq n\right\} \cup\{0\}$ with multiplication rule $e_{i j} e_{k l}=\delta_{j k} e_{i l}$. Note that $S$ has the monoid spine $\left\{e_{11}, \ldots, e_{n n}\right\} \cup\{0\}$.

Theorem 2.17. Assume that $S$ is a multiplicative monoid (with or without zero) and $T$ is a monoid spine of $S$. Assume furthermore that $A$ is a semiring and $N$ is an additive spine of $A$. Then $N \cdot T$ is an additive spine of $A[S]$.

Proof. Let $R:=A[S]$ and $R_{1}:=C[S] \subset R$, with $C$ the image of the (unique) homomorphism $\mathbb{N}_{0} \rightarrow A$. It is obvious that $R_{1}=\sum^{\infty} S$ and that $S$ is contained in the halo $\widetilde{T}$ of $T$ in $R_{1}$. Thus $T$ is a monoid spine of $R_{1}$. (In fact it can be verified that $\widetilde{T}=\widetilde{S}=S$.) Let $R_{2}:=A \subset R$. 
Then $R=\sum^{\infty} R_{1} \cdot R_{2}$ and the elements of $R_{1}$ commute with those of $R_{2}$. The assertion follows from Proposition 2.12.

\section{Halos AND ADDitive SPINES in $R$-MOdules}

In the following $V$ is again an $R$-module.

Example 3.1. If $S$ is a set of generators of the $R$-module $V$ and $M$ is an additive spine of $R$, then we know by Theorem 2.8 that $M S$ is an additive spine of $V$.

Theorem 2.8 generalizes as follows:

Theorem 3.2. Assume that $S$ is an additive spine of an $R$-module $V$. Then every $S A$ submodule $W$ of $V$ is generated by $W \cap S$, and moreover $W \cap S$ is an additive spine of $W$.

Proof. a) We first verify that $V$ itself is generated by $S$. Since $V$ is additively generated by $\widetilde{S}$, for given nonzero $v \in V$ we have

$$
v=\sum_{i=1}^{n} v_{i},
$$

with $n \in \mathbb{N}, v_{i} \in \widetilde{S}$. There exist $\lambda_{i}, \mu_{i} \in R$ such that

$$
\begin{gathered}
s_{i}:=\lambda_{i} v_{i} \in S, \\
v_{i}=\mu_{i} s_{i},
\end{gathered}
$$

and so by (A)

$$
v=\sum_{i=1}^{n} \mu_{i} s_{i}
$$

and we are done.

b) If now $W$ is an SA-submodule of $V$, and the above element $v$ lies in $W$, then in Equation (A) all summands $v_{i}$ are in $W$, and so the $s_{i}$ from $(\mathrm{B})$ are in $W \cap S$. We conclude from (B) and (C) that all $v_{i}$ are in the halo $(W \cap S)^{\sim}$ of $W \cap S$ in $W$, and we infer from (A) that $W$ is additively generated by $(W \cap S)^{\sim}$, i.e., $W \cap S$ is an additive spine of $W$. As proved in a) the set $W \cap S$ generates the $R$-module $W$.

We write down a chain of propositions which turn out to be useful in working with halos and additive spines. For clarity we sometimes denote the halo of a set $S$ in $V$ more elaborately by $\operatorname{hal}_{V}(S)$ instead of $\widetilde{S}$.

Proposition 3.3. If $S$ is a subset of an $R$-module $V$ and $W$ a submodule of $V$, then

$$
W \cap \operatorname{hal}_{V}(S)=\operatorname{hal}_{W}(W \cap S)=\operatorname{hal}_{V}(W \cap S) .
$$

Proof. Let $v \in \operatorname{hal}_{V}(S)$ be given. We choose $\lambda, \mu \in R$ with $\lambda v=s \in S$ and $\mu s=v$. If now $v \in W$ then $\lambda v=s \in W \cap S$, and so $v \in \operatorname{hal}_{W}(W \cap S)$. This proves that

$$
W \cap \operatorname{hal}_{V}(S) \subset \operatorname{hal}_{W}(W \cap S) .
$$

Trivially

$$
\operatorname{hal}_{W}(W \cap S) \subset \operatorname{hal}_{V}(W \cap S) .
$$

If $v \in \operatorname{hal}_{V}(W \cap S)$, then there exist $\lambda, \mu \in R$ with $\lambda v=s \in W \cap S$ and $\mu s=v$. It follows that $v \in W \cap \operatorname{hal}_{V}(S)$. This proves

$$
\operatorname{hal}_{V}(W \cap S) \subset W \cap \operatorname{hal}_{V}(S) .
$$

(A) - (C) together imply the assertion of the proposition. 
In case $S \subset W$ the proposition reads as follows:

Corollary 3.4. Let $S \subset V$. Then the halo of $S$ in any submodule $W \supset S$ of $V$ coincides with the halo of $S$ in $V$.

Thus in practice the notation $\operatorname{hal}_{V}(S)$ instead of $\widetilde{S}$ is rarely needed.

Proposition 3.5. Let $\left(V_{i} \mid i \in I\right)$ be a family of submodules of the $R$-module $V$ and assume that for every $i \in I$ there is given a set $S_{i} \subset V_{i}$.

a) Then

$$
\bigcup_{i \in I} \operatorname{hal}_{V_{i}}\left(S_{i}\right)=\operatorname{hal}_{V}\left(\bigcup_{i \in I} S_{i}\right) .
$$

b) If $\sum_{i \in I} V_{i}=V$ and each $S_{i}$ is an additive spine of $V_{i}$, then $\bigcup_{i \in I} S_{i}$ is an additive spine of $V$.

Proof. Let $S:=\bigcup_{i \in I} S_{i}$.

a): We have $\operatorname{hal}_{V}(S)=\bigcup_{i \in I} \operatorname{hal}_{V}\left(S_{i}\right)$ in complete analogy to Remark 2.2,iii. Furthermore $\operatorname{hal}_{V}\left(S_{i}\right)=\operatorname{hal}_{V_{i}}\left(S_{i}\right)$ by Corollary 3.4 ,

b): Let $\widetilde{S}_{i}:=\operatorname{hal}_{V_{i}}\left(S_{i}\right)$. Then $\bigcup_{i \in I} \widetilde{S}_{i}=\widetilde{S}, \sum^{\infty} \widetilde{S}_{i}=V_{i}$, and so

$$
\sum^{\infty} \widetilde{S}=\sum_{i \in I}\left(\sum^{\infty} \widetilde{S}_{i}\right)=\sum_{i \in I} V_{i}=V .
$$

We now have a good hold on all additive spines of a free $R$-module as follows:

Proposition 3.6. Assume that $V$ is a free $R$-module with base $\left(v_{i} \mid i \in I\right)$. Then every additive spine $S$ of $V$ has the form

$$
S=\bigcup_{i \in I} M_{i} v_{i}
$$

where every $M_{i}$ is an additive spine of $R$, as defined in 9 .

Proof. We have $V=\bigoplus_{i \in I} V_{i}$ with $V_{i}=R v_{i} \cong R$. The claim follows from Proposition 3.5.

Proposition 3.7 (Functoriality of halos and additive spines). Let $\varphi: V \rightarrow V^{\prime}$ be an $R$-linear map between $R$-modules.

a) If $S$ is a subset of $V$, then

$$
\varphi(\widetilde{S}) \subset \varphi(S)^{\sim}
$$

b) If $\underset{\widetilde{S}}{S}$ is an additive spine of $V$, then the $R$-module $\varphi(V)$ is additively generated by $\varphi(\widetilde{S})$, and so $\varphi(S)$ is an additive spine of $\varphi(V)$.

Proof. a): Let $x \in \widetilde{S}$. We have $\lambda, \mu \in R$ with $\lambda x=s \in S, \mu s=x$. It follows that $\lambda \varphi(x)=\varphi(s), \mu \varphi(s)=\varphi(x)$, whence $\varphi(x) \in \varphi(S)^{\sim}$.

b): By Corollary 3.4 we may replace $V$ by $\varphi(V)$, and so assume that $\varphi$ is surjective. We have $\sum^{\infty} \widetilde{S}=V$. Applying $\varphi$, we obtain

$$
\sum^{\infty} \varphi(\widetilde{S})=\varphi(V) .
$$


It follows by a) that $\sum^{\infty} \varphi(S)^{\sim}=\varphi(V)$.

Corollary 3.8. Assume that $R$ and $T$ are semirings and $V$ is an $(R, T)$-bimodule, i.e., $V$ is a left $R$-module, a right $T$-module, and

$$
\forall \lambda \in R, \mu \in T, v \in V:(\lambda v) \mu=\lambda(v \mu) .
$$

Let $S$ be a subset of $V$. As before let $\widetilde{S}$ denote the halo of $S$ in ${ }_{R} V$ (which means $V$ as a left $R$-module). Then, for any $t \in T$

$$
\widetilde{S} t \subset(S t)^{\sim} .
$$

If $S$ is an additive spine of $V$, then $\widetilde{S} t$ generates the left $R$-module $V t$ additively, and so $S t$ is an additive spine of $V t$.

Proof. Apply Proposition 3.7 to the endomorphism $v \mapsto v t$ of ${ }_{R} V$.

Corollary 3.9. If again $V$ is an $(R, T)$-bimodule and $t$ is a unit of $T$, then $\widetilde{S} t=(S t)^{\sim}$, and $S$ is an additive spine of $V$ iff $S t$ is an additive spine of $V$.

Proof. Let $u:=t^{-1}$. Then by Corollary $3.8(\widetilde{S} t) u \subset(S t)^{\sim} u \subset(S t u)^{\sim}=\widetilde{S}$. Multiplying by $t$, we obtain $\widetilde{S} t \subset(S t)^{\sim} \subset \widetilde{S} t$, whence $\widetilde{S} t=(S t)^{\sim}$, and then

$$
\sum^{\infty}(S t)^{\sim}=\left(\sum^{\infty} \widetilde{S}\right) t
$$

Example 3.10. $R$ is an $(R, R)$-bimodule in the obvious way. Thus, if $M$ is an additive spine of $R$ and if $u$ is a unit of $R$, then $M u$ is again an additive spine of $R$.

Example 3.11. Assume that $C$ is a semiring that is a homomorphic image of $\mathbb{N}_{0}$, and $R:=M_{n}(C)$. We have seen in Example 2.12 that $\left\{e_{11}, \ldots, e_{n n}\right\}$ is an additive spine of $R$. Let $\sigma \in S_{n}$. Then $u:=\sum_{i=1}^{n} e_{i \sigma_{(i)}}$ is a unit of $R$, namely $u$ is the permutation matrix of $\sigma^{-1}$. We have $e_{i i} u=e_{i \sigma(i)}$, and conclude that $\left\{e_{1 \sigma(1)}, \ldots, e_{n \sigma(n)}\right\}$ is an additive spine of $M_{n}(C)$.

We can generalize Proposition 2.12 as follows:

Proposition 3.12. Assume that $R_{1}, R_{2}$ are commuting subsemirings of a semiring $R$ with $R=\sum^{\infty} R_{1} R_{2}$, and that $V_{1}, V_{2}$ are left modules over $R_{1}$ and $R_{2}$ respectively. Assume furthermore that there is given a composition $V_{1} \times V_{2} \stackrel{\bullet}{\longrightarrow}$ such that

$$
\left(\lambda_{1} \lambda_{2}\right)\left(v_{1} \bullet v_{2}\right)=\left(\lambda_{1} v_{1}\right) \bullet\left(\lambda_{2} v_{2}\right)
$$

for any $\lambda_{i} \in R, v_{i} \in V_{i}(i=1,2)$. Assume finally that $V=\sum^{\infty} V_{1} \bullet V_{2}$. Then, given subsets $S_{i} \subset V_{i}$ with halos $\widetilde{S}_{i}$ in the $R_{i}$-module $V_{i}(i=1,2)$, the following holds.

a) $\widetilde{S}_{1} \bullet \widetilde{S}_{2}$ is contained in the halo $\left(S_{1} \bullet S_{2}\right) \sim$ of $S_{1} \bullet S_{2}$ in $V$.

b) If $S_{i}$ is an additive spine of $V_{i}(i=1,2)$, then

$$
V=\sum^{\infty} \widetilde{S}_{1} \bullet \widetilde{S}_{2}
$$

and $S_{1} \bullet S_{2}$ is an additive spine of $V$.

Proof. Let $v_{i} \in \widetilde{S}_{i}(i=1,2)$. We have $\lambda_{i}, \mu_{i} \in R_{i}$ with $\lambda_{i} v_{i}=s_{i} \in S_{i}, \mu_{i} s_{i}=v_{i}$. Now

$$
\left(\lambda_{1} \lambda_{2}\right)\left(v_{1} \bullet v_{2}\right)=\left(\lambda_{1} v_{1}\right) \bullet\left(\lambda_{2} v_{2}\right)=s_{1} \bullet s_{2}
$$


and $\left(\mu_{1} \mu_{2}\right)\left(s_{1} \bullet s_{2}\right)=\left(\mu_{1} s_{1}\right) \bullet\left(\mu_{2} s_{2}\right)=v_{1} \bullet v_{2}$. This proves that $\widetilde{S}_{1} \bullet \widetilde{S}_{2} \subset\left(S_{1} \bullet S_{2}\right) \sim$. If now $\sum^{\infty} \widetilde{S}_{i}=V_{i}(i=1,2)$, then

$$
\sum^{\infty}\left(\widetilde{S}_{1} \bullet \widetilde{S}_{2}\right) \supset\left(\sum^{\infty} \widetilde{S}_{1}\right) \bullet\left(\sum^{\infty} \widetilde{S}_{2}\right)=V_{1} \bullet V_{2},
$$

and so $\sum^{\infty}\left(\widetilde{S}_{1} \bullet \widetilde{S}_{2}\right) \supset \sum^{\infty} V_{1} \bullet V_{2}=V$, whence $\sum^{\infty} \widetilde{S}_{1} \bullet \widetilde{S}_{2}=V$. A fortiori $\sum^{\infty}\left(S_{1} \bullet S_{2}\right)^{\sim}=V$.

Note that Proposition 2.12 is indeed a special case of this proposition: Given an $R$ module $V$, take $R_{1}=R_{2}=R, V_{1}=R, V_{2}=V$ and the scalar product $R \times V \rightarrow V$.

\section{The posets $\mathrm{SA}(V), \Sigma \mathrm{SA}(V)$ And $\Sigma_{f} \mathrm{SA}_{f}$ IN GOOd CASES}

Assume that $R$ has a finite additive spine $M$ consisting of $m:=|M|$ elements. We have seen in $\$ 2$ that, when $S$ is a set of generators of $V$, then every $W \in \mathrm{SA}(V)$ is generated by the set $W \cap(M S)$. Thus, if $s:=|S|$ is finite, we see that the lattice $\operatorname{SA}(V)$ is finite, consisting of at most $2^{m|S|}$ elements. More generally we have the following fact.

Theorem 4.1. Assume that $V_{0}$ is a submodule of an $R$-module $V$ and $S$ is a subset of $V$, such that $V$ is generated over $V_{0}$ by $S$, i.e.,

$$
V=V_{0}+\sum^{\infty} R S
$$

Assume that $R$ has a finite additive spine $M$ consisting of $m:=|M|$ elements.

Let $W_{0} \in \mathrm{SA}(V)$ be given with $W_{0} \subset V_{0}$, and consider the set

$$
\mathrm{SA}\left(V ; W_{0}, V_{0}\right):=\left\{W \in \mathrm{SA}(V) \mid W \cap V_{0}=W_{0}\right\} .
$$

Then, if $s:=|S|$ is finite, this set $\mathrm{SA}\left(V ; W_{0}, V_{0}\right)$ consists of at most $2^{\text {ms }}$ elements. Furthermore, any chain $W_{0} \varsubsetneqq W_{1} \varsubsetneqq \cdots \varsubsetneqq W_{r}$ in $\mathrm{SA}\left(V ; W_{0}, V_{0}\right)$ has length $r \leq m s$.

Proof. Let $U$ denote the submodule of $V$ generated by $S$. We have $V=V_{0}+U$. If $W \in$ $\mathrm{SA}\left(V ; W_{0}, V_{0}\right)$, then by $(1.1)$

$$
W=W \cap V_{0}+W \cap U=W_{0}+W \cap U,
$$

and, of course, $W \cap U \in \mathrm{SA}(U)$. Since $|\mathrm{SA}(U)| \leq 2^{m s}$, as stated above, we infer that $\left|\mathrm{SA}\left(V ; W_{0}, V_{0}\right)\right| \leq 2^{m s}$. Also, if $W_{0} \varsubsetneqq W_{1} \varsubsetneqq \cdots \varsubsetneqq W_{r}$ is a chain in $\operatorname{SA}\left(V ; W_{0}, V_{0}\right)$, we conclude from (4.3) for $U_{i}:=W_{i} \cap U$ that

$$
U_{0} \varsubsetneqq U_{1} \varsubsetneqq \cdots \varsubsetneqq U_{r}
$$

Every $U_{i}$ is generated by the set $U_{i} \cap(M S)$ and so

$$
U_{0} \cap(M S) \varsubsetneqq U_{1} \cap(M S) \varsubsetneqq \cdots \varsubsetneqq U_{r} \cap(M S) .
$$

This implies that $r \leq|M S|=m s$.

We return to an arbitrary semiring $R$ and permit infinite sums of SA-submodules, writing $U \in \Sigma \mathrm{SA}(V)$, called a $\Sigma \mathrm{SA}$-submodule of $V$.

Theorem 4.2. Assume that $T$ is an additive spine of the $R$-module $V$ (cf. Definition 1.2).

a) Then any $U \in \Sigma \mathrm{SA}(V)$ is generated by the set $U \cap T$.

b) $|T|=t$ is finite, then $|\Sigma \mathrm{SA}(V)| \leq 2^{t}$, and any chain

$$
U_{0} \varsubsetneqq U_{1} \varsubsetneqq \cdots \varsubsetneqq U_{r}
$$

in $\Sigma \mathrm{SA}(V)$ has length $r \leq t$. 
Proof. a): Write $U=\sum_{i \in I} W_{i}$ with $W_{i} \in \mathrm{SA}(V)$. We know by Theorem 3.2 that every $W_{i}$ is generated by $W_{i} \cap T$. Thus $U$ is generated by the set

$$
\bigcup_{i \in I}\left(W_{i} \cap T\right)=\left(\bigcup_{i \in I} W_{i}\right) \cap T .
$$

A fortiori $U$ is generated by $U \cap T$.

b): Every $U \in \Sigma \mathrm{SA}(V)$ is generated by the set $U \cap T \subset T$. We have at most $2^{t}$ possibilities for this set, and so $|\Sigma \mathrm{SA}(V)| \leq 2^{t}$. Furthermore, if $U_{0} \varsubsetneqq \cdots \varsubsetneqq U_{r}$ is a chain in $\Sigma \mathrm{SA}(V)$, then

$$
U_{0} \cap T \varsubsetneqq U_{1} \cap T \varsubsetneqq \cdots \varsubsetneqq U_{r} \cap T
$$

since each $U_{i}$ generated by $U_{i} \cap T$, and so $r \leq t$.

We denote the set of all finitely generated SA-submodules of $V$ by $\operatorname{SA}_{f}(V)$, and the set of submodules of $V$, which are sums of finitely many elements of $\mathrm{SA}_{f}(V)$, by $\Sigma_{f} \mathrm{SA}_{f}(V)$. (Note that a module in $\Sigma_{f} \mathrm{SA}_{f}(V)$ is finitely generated, but perhaps not $\mathrm{SA}$ in $V$.)

By a variation of our previous arguments we obtain

Theorem 4.3. Assume that $R$ has a finite additive spine $M$, and also that $U \in \Sigma_{f} \operatorname{SA}_{f}(V)$. Let $S$ be a finite set of generators of $U$. Then every $W \in \mathrm{SA}_{f}(U)$ is generated by the finite set $W \cap(M S)$ and every chain

$$
W \supsetneqq W_{1} \supsetneqq W_{2} \supsetneqq \cdots \supsetneqq W_{r}
$$

in $\mathrm{SA}(U)$, hence in $\mathrm{SA}_{f}(U)$, has length $r \leq|M| \cdot|S|$. A fortiori this holds if $W$ and all $W_{i}$ are in $\mathrm{SA}_{f}(V)$.

Proof. Every $W \in \mathrm{SA}_{f}(U)$ is generated by the finite set $W \cap(M S)$, cf. Theorem 2.8. Furthermore, by the same theorem, every $W_{i}$ is generated by the subset $W_{i} \cap(M S)$ of $W \cap(M S)$. It follows that

$$
W \cap(M S) \supsetneqq W_{1} \cap(M S) \supsetneqq \cdots \supsetneqq W_{r} \cap(M S)
$$

and so $r \leq|W \cap(M S)| \leq|M| \cdot|S|$. It is obvious that every SA-submodule of $V$ contained in $U$ is $\mathrm{SA}$ in $U$.

Our final result refers to modules with additive spines which are not necessarily finite.

Theorem 4.4. Assume that $T \subset V$ is an additive spine of the $R$-module $V$, and $U \in \Sigma \mathrm{SA}(V)$.

a) Then $U$ is generated by the set $U \cap T$.

b) If $U$ is an $\mathrm{SA}_{f}$-sum in $V$, and $\left(W_{i} \mid i \in I\right)$ is a family of finitely generated SAsubmodules of $V$ with $U=\sum_{i \in I} W_{i}$, then every $W_{i}$ is generated by a finite subset $T_{i}$ of $W_{i} \cap T$, and so $U$ is generated by the subset $\bigcup_{i \in I} T_{i}=T^{\prime}$ of $T$. This subset $T^{\prime}$ is an additive spine of $U$.

c) If $U \in \Sigma_{f} \mathrm{SA}_{f}(V)$ then $U$ is generated by a finite subset of $U \cap T$, and this is an additive spine of $U$.

Proof. We choose a family $\left(W_{i} \mid i \in I\right)$ in $\operatorname{SA}_{f}(V)$ with $U=\sum_{i \in I} W_{i}$.

a): Done before (Theorem 4.2).

b): We assume now that all $W_{i}$ are finitely generated. Every $W_{i}$ is generated by $W_{i} \cap T$ (Theorem 3.2). It follows that $W_{i}$ is generated by a finite subset $T_{i}$ of $W_{i} \cap T$. Indeed, given generators $s_{1}, \ldots, s_{r}$ of $W_{i}$ for $i$ fixed, write every $s_{j}$ as a linear combination of a finite 
subset $T_{i j}$ of $W_{i} \cap T$. Then $T_{i}:=\bigcup_{j=1}^{r} T_{i j}$ does it. It follows by Theorem 3.2 that $T_{i}$ is an additive spine of $W_{i}$. It now is clear that $T^{\prime}:=\bigcup_{i \in I} T_{i}$ generates $U=\sum_{i \in I} W_{i}$, and it follows by Proposition 3.5 that $T^{\prime}$ is an additive spine of $U$.

c): Now evident, since the index set $I$ can be assumed to be finite, and so $T^{\prime}=\bigcup_{i \in I} T_{i}$ is a finite additive spine of $U$.

\section{REFERENCES}

[1] A.A. Costa. Sur la thêorie générale des demi-anneaux, Publ. Math. Decebren 10:14-29, 1963.

[2] J. Golan. Semirings and their Applications, Springer-Science + Business, Dordrecht, 1999. (Originally published by Kluwer Acad. Publ., 1999.)

[3] Z. Izhakian, M. Johnson, and M. Kambites. Pure dimension and projectivity of tropical convex sets, Adv. in Math., 303:1236-1263, 2016.

[4] Z. Izhakian, M. Knebusch, and L. Rowen. Decompositions of modules lacking zero sums. Israel J. Math., 225(2):503-524, 2018.

[5] Z. Izhakian, M. Knebusch, and L. Rowen. Summand absorbing submodules, J. Pure and Appl. Alg., to appear.

Institute of Mathematics, University of Aberdeen, AB24 3UE, Aberdeen, UK.

E-mail address: zzur@abdn.ac.uk

Department of Mathematics, NWF-I Mathematik, Universität Regensburg 93040 RegensBURG, GERMANY

E-mail address: manfred.knebusch@mathematik.uni-regensburg.de

Department of Mathematics, Bar-Ilan University, 52900 Ramat-Gan, Israel

E-mail address: rowen@math.biu.ac.il 